\title{
How Are You Feeling?
}

\section{A Social Network Model to Monitor the Health of Post-Operative Patients}

\author{
James J. Mulcahy \\ Department of Electrical and \\ Computer Engineering and \\ Computer Science \\ Florida Atlantic University \\ Boca Raton, FL, USA \\ jmulcah1@fau.edu
}

\author{
Shihong Huang \\ Department of Electrical and \\ Computer Engineering and \\ Computer Science \\ Florida Atlantic University \\ Boca Raton, FL, USA \\ shihong@fau.edu
}

\author{
Junwei Cao \\ Research Institute of \\ Information Technology \\ Tsinghua University \\ Beijing, China \\ caoj@mit.edu
}

\author{
Fan Zhang \\ Research Institute of \\ Information Technology \\ Tsinghua University \\ Beijing, China \\ zhang- \\ fan07@mails.tsinghua.edu.cn
}

\begin{abstract}
Mobile devices and cloud computing platforms have become increasing popular in recent years, as more ways in which to adopt these technologies to existing business and research models have been envisioned and implemented. What started as simply providing bandwidth and technology to mobile users in order to browse the internet from handheld devices now allows entire websites to be hosted on remote cloud platforms. Users now have the ability to trade and track stock market data from anywhere and anytime, automatically communicate data from wearable medical sensor devices, avoid vehicle collisions, track freight shipments, and a plethora of other applications.
\end{abstract}

Healthcare is an area where the leveraging of these new technologies has the ability to have worldwide impact, with many exciting possibilities. However, much of the current focus is on more "doctor-centric" uses rather than "patient-centric" ones. The use of mobile devices to automatically sense and transmit data from the patient, devices to aid in dictation and communication within hospital settings, and cloud products to store patient data and other solutions help make the tasks of physicians easier, but do not necessarily improve the care given to the patient. There are bandwidth concerns, security concerns and the general cyberphobia that consumers commonly have with sharing personal information. This paper proposes a different type of solution that is more related to a social networking environment in which many people are already comfortable using. It concentrates on the collection of data from patients during a period of time that they are often suffering the most - after initial post-operative release from a hospital or clinic. It first explores technologies that are currently in use, and then describes a different model that allows the patient to control what information is being shared, who is allowed to consume this data, and how it might enhance the well-being of those that may make use of this new paradigm.

Keywords: social networking, mobile computing, healthcare, cloud computing, modeling, patient-centric, monitoring, simulation

This material is based upon work supported by the National Science Foundation under Grant No. OISE-0730065.

\section{INTRODUCTION}

\section{A. Shift In Technology}

Modern computing technology has shifted from the classic localized and client-server models to mobile and cloud-based platforms. Business and research sectors continue to discover ways to leverage these new approaches. At first, the most obvious applications enabled mobile users to send and check email, browse websites, play games, and other activities that were once done via desktop or laptop computers [1][2]. Other less trivial implementations followed that resulted in an explosion of device-specific applications that allowed users to do everything from tracking and controlling financial information to monitoring caloric intake and arming security systems in their homes from remote locations [3]. Commercially, mobile devices changed how companies inventoried and monitored their products, how shipping companies scanned and reported package pickups, and even how meal orders in some restaurants were recorded and delivered to the kitchen staff [4][5]. More sophisticated devices emerged that enabled government and military authorities to monitor and track persons domestically and on the battlefield [6]. Vehicles became mobile ad hoc networks that were able to guide drivers to their destinations, avoid collisions with other vehicles, and sense road conditions, among other advantages [7].

\section{B. Applications In Healthcare}

Medical applications have allowed health care industries to find ways to make use of mobile technology. Devices inside hospital environments allow physicians and supporting staff to communicate with each other [8], to monitor patients, store and transmit electronic medical records (EMRs), and to replace older methods of dictation, once accomplished manually by translation from voice recordings [9]. Implantable and wearable devices have been developed to monitor glucose levels in diabetic patients and heart data in cardiology patients [10]. Telemedicine now allows patients in remote areas to have x-rays, MRIs and other diagnostic tests performed at sites closer their homes, with the data transmitted to physicians that are hundreds, even thousands of miles away 
[11]. Remote diagnosis and health-care advice can be administered to rural and disaster areas in this manner. The general public and physicians alike can now access informative websites such as WebMD to obtain information about specific medications and their interactions [12].

\section{Potential for Improvement}

In healthcare, much focus has been placed on making the work of physicians or medical facilities more efficient with automated processes and use of modern hardware and software techniques. However some subjective areas that directly relate to the well-being of patients are largely ignored or underdeveloped using new technologies. For example, in nursing, specific details about how nurses apply their practices is an area that has recently begun to receive more attention [13][14].

One area that seems to be glaringly underdeveloped using new technologies is the monitoring of patients that have had recent surgical or medical procedures. Typically, a patient that is discharged from a hospital or doctor's office is sent home with instructions from the doctor related to wound care, medications and at-home rehabilitation, if appropriate. One or more post-operative appointments with the physician are often scheduled for periods of several days to several weeks after initial discharge. At these visits, the patient typically consults with the physician about their progress, problems they encountered, and future needs.

The rest of this paper is organized as follows: Section 2 further explores the topic of post-operative patient care. Section 3 proposes a solution that uses mobile and cloud technology using a social networking model. Section 4 identifies the advantages and challenges of this solution, while Section 5 describes current and future work towards this direction. The paper is concluded in section 6 and remarks on the efficacy of the proposed solution.

\section{POST-OPERATIVE PATIENT CARE}

Patient care after discharge from a hospital or clinic can require varying degrees of monitoring by their physicians. In more serious cases, a physician may need to actively monitor the well-being of the patient. This is sometimes accomplished with an in-home nurse or medical staff that regularly visits the patient, which can be very expensive. In other situations, communication between doctor and patient or the patient's caregivers is accomplished via conventional methods such as the telephone. Most of the time, the patient is simply sent home with specific instructions for wound care, medication regimen and physical therapy instructions (if needed), and expected to return days or weeks later for a follow-up visit with the doctor.

It is this latter case that is the focus of this paper and of the solution being proposed. It is not uncommon for a patient to leave the hospital after surgery and have no contact with a physician for the entire period between discharge and the scheduled follow-up appointment. During this time, the patient may experience pain and discomfort, ingest too much or too little medication, experience swelling or infection of surgical wounds or any number of other conditions that may or may not warrant contacting the doctor. Since most patients are not medical professionals themselves, they may have difficulty recognizing when symptoms are serious enough to contact the physician or facility from which they are discharged. They may, in fact, suffer to varying degrees until they return for their post-operative appointment. In more serious cases, patients may develop infections or serious drug interaction problems that progress to a dangerously critical condition before they are convinced of the need to contact medical staff. Of course, contacting a physician can itself be a challenge. A patient's first contact may be with an "on-call" service that notes their contact information and then relays the information to the appropriate physician, and the patient waits for a return call.

In some cases, a patient may experience less critical problems such as swelling, insomnia, nausea, or drug interaction issues that may not be brought to the attention to the physician for days or weeks, if at all. It is in this area that we focus our work. What if the patient was able to use mobile technology to update "how they are feeling?" This kind of data could be collected and stored in a centralized location, intermittently or constantly monitored by medical staff. The data could also serve as a sort of "diary" for the patient, accessible by that patient so they can subjectively monitor their own progress. The collection of updates could also be gathered by medical staff so that by the time the patient arrives for a follow-up appointment, the doctor already has reviewed the symptoms, complaints, pain levels and any other data the patient has been providing. This enables the physician to have such information "in hand" at the time of the appointment. Some of the finer details may be forgotten by the patient by this time, and a log of this type could prove to be more educational for both the patient and physician, especially if it has been collected over the entire period between discharge and followup. After all, a patient may not be able to objectively compare their pain level the day after coming home from a hospital for a surgical procedure with the pain level they felt five, ten or fourteen days later. What if the patient were able to continually update their condition in a social network setting, much like we use Twitter, Facebook, or Myspace today to do the same?

\section{A MOBILE CLOUD SOLUTION FOR PATIENTS}

\section{A. Software Solution for Patients}

The solution proposed by this paper is to establish a cloudbased data storage and website that is accessible via desktop, laptop or mobile device. Access to the website would be using a simple login procedure, with a User ID and password unique to the patient, a method already in use in other social network environments. Once logged in, a patient first chooses from a list of "medical events" that the patient can create. Patients that have had more than one medical event would likely choose the most recent event (e.g. "ACL knee reconstruction"). The patient can then choose from a list of options designating the type of data they are about to supply. For example, a patient 
could choose "Pain Level" to record their current (or past) pain level from a predetermined scale that is defined by the website.

A simple session under this scenario might be described by the steps shown in Table I. The implemented software system could contain more or fewer options. Some options could cause other sub-options to appear (e.g. choosing a medication type could cause another option to choose dosage to appear).

TABLE I. Sample User Session

\begin{tabular}{|l|l|}
\hline User Operation & User Entry \\
\hline Logs in & Provides UserID \& Password \\
\hline Chooses Medical Event & ACL Reconstruction (7/2010) \\
\hline Chooses Data Type & Pain Level \\
\hline Chooses Pain Level & 3 (Bearable but noticeable) \\
\hline Chooses Data Type & Medication \\
\hline Chooses Medicine Type & Oxycodone \\
\hline Chooses Medicine Dosage & $10 / 500$ \\
\hline Chooses Data Type & Wound Maintenance \\
\hline Choose Maintenance Location & Ice \\
\hline Chooses Wound Location & Left Knee \\
\hline Logs out & Confirms Logout \\
\hline
\end{tabular}

Of course a patient does not necessarily log out, and may choose to stay connected to the session. This is especially true if they are logged in using a mobile device that uses an application that automatically handles the login and logout process, or the patient does not close a website page when connected using a desktop or laptop.

Various types of data could be collected in this manner. While not intended to be a comprehensive list, some examples of the type of details the implementation could collect include:

1. Event management (procedure type and date)

2. Medication management (type and dosage)

3. Wound management (icing or re-dressing)

4. Activity/Therapy (walking or driving)

5. Symptoms (nausea or soreness)

6. Comments (free-form entry of information)

In each case, the patient would have the option of choosing whether the data being input is valid "right now", or could optionally specify an earlier time or date. In some cases, a patient may take medication at a certain time, but does not update the system to reflect this until later. Data entry detail and frequency would be, of course, completely voluntary and at the discretion of the patient.

An example of a log patient might generate is shown in Table II. The content can have a very high level of detail, or a much shallower one, depending on the patient. The focus of the proposed solution is to create a system that is easy to use, with as simple a user interface as possible. Most importantly, all information supplied is at the sole discretion of the patient. Perhaps all that a patient prefers to be recorded are medication dosage details in order to keep track of such information to avoid forgetting to take medication or duplicating a dosage.
TABLE II. Sample User Activity Log

\begin{tabular}{|c|l|ll|}
\hline \multicolumn{2}{|c|}{ Patient: 18392642389} & \multicolumn{1}{l|}{ Date: August $17^{\text {th }}, 2010$} \\
\hline DATE & TIME : ACL reconstruction surgery (07/29/10) \\
\hline $07 / 30 / 2010$ & $05: 00$ & Commentary: & Discharge \\
\hline $07 / 30 / 2010$ & $05: 00$ & Pain Level: & 8 \\
\hline $07 / 30 / 2010$ & $07: 10$ & Commentary: & Home \\
\hline $07 / 30 / 2010$ & $07: 14$ & Pain Level: & 9 \\
\hline $07 / 30 / 2010$ & $07: 20$ & Medication: & Oxycodone \\
\hline $07 / 30 / 2010$ & $07: 22$ & Wound Mgmt: & Ice left knee \\
\hline $07 / 30 / 2010$ & $07: 45$ & Pain Level: & 6 \\
\hline
\end{tabular}

\section{B. Who Consumes The Data?}

The novelty of this type of data collection is that the frequency and detail level of its use can be flexible, and dictated completely by the patient. A patient using this type of system can use it as a personal medical diary, recording their experiences and progress, and having sole access to that data. A future medical event of the same type could allow the patient to review previous data and make informed decisions based upon prior experiences. A patient could also choose to print or record the resulting data and bring it to their next medical consultation. This type of information would be valuable in the ability of physicians to understand the impact of their postoperative instructions and medicines prescribed, or cause them to modify or more closely monitor patients between the current and subsequent post-operative visits. Perhaps the physician notes elevated pain levels and adjusts a patient's medications, or revises instructions related to wound management or physical activity.

The patient could also opt for medical staff to have access to their data by adding the physician's own User ID to a "white list" of allowed data accessors. The physician could subsequently log into the same system with their own unique User ID and access the patient data. In the proposed system, the physician would not see the patient's name or User ID, but rather a system-generated unique user identification number (UIN) that the patient would provide to the doctor through conventional means (verbally, in person, by mail, e-mail, etc). Note that this technique has some desirable advantages related how people choose to share or not share their medical information: 1) the patient completely controls who sees and does not see their data, 2) only the patient contributes to the data, not the physician or third parties, and 3) no specific identifiable patient information is ever stored in the cloud database (real name, date of birth, health care provider, etc). Any association with between the UIN and the actual identification of a particular patient would be maintained by the physician in their own facility, thereby completely removing the issue of privacy from this cloud-based implementation.

With approved access to the patient's data, a physician could choose to actively or intermittently monitor the updates by the patient during the period between appointments, or choose to view or print the data in advance of the patient's next 
visit in order to have "in hand" a detailed report of the patient's experience since the physician last saw him or her. This could have an effect on what type of topics the physician discusses with the patient, and what kind of actions are to be taken based on the data. It could even affect how the physician treats the same or future patients in a similar context or with similar surgical procedures. Note that the physician is only a consumer of this data, and does not have the ability to alter or directly augment it.

Another exciting possibility for this type of data-logging and sharing is that a patient could choose to anonymously allow their data to be mined by third parties that are interested in collecting data about particular facilities, physicians, procedures, medications and a myriad of other potential research areas. For example, a researcher may want to measure the pain levels of all knee surgery patients using a particular medication, or correlate physical therapy to pain levels, and so on. This type of data mining could prove to be useful in all areas of healthcare depending on the participation and level of adoption of the proposed solution.

\section{ADVANTAGES AND CHALLENGES}

\section{A. Advantages}

This solution has the potential to allow the collection and use of data that has not been examined before. What was once expressed verbally between patient and physician can now be recorded and analyzed in a much more structured and perhaps automated manner. A patient seeing a physician fourteen days after a major surgery, for example, may not remember what their pain level that day is compared to how they felt two days after surgery. If they did not keep written track of medication dosage, they may not remember to mention to the physician that they stopped taking medication because of an interaction with other medicines, or that they did not follow the prescribed dosage amounts. This is all information that is currently invisible to the physician and therefore potentially valuable knowledge.

The proposed solution leverages existing technology without inventing any new hardware, software techniques or security procedures. Cloud-based computing is already proven to be a viable way of storing large amounts of data in a homogenous way and hosting websites, some that are accessible more than $99.9 \%$ of the time [15]. Mobile computing is already prevalent around the world, with more than 4.6 billion cellular accounts in 2010 [16], compared to approximately 6.8 billion in world population [17].

The positive impact on rural areas of large countries such as China and India could prove to be extraordinary, as well as to all corners of the world where the well-being of patients are not actively monitored between hospital discharge and the patient's next contact with medical personnel.

Issues related to pain, drug interactions, wound management and physical therapy could be collected, leading to new approaches developed using conclusions from data patterns not previously recognized due to the lack of communication between patient and physician during the time between post-operative visits.

\section{B. Challenges}

Who pays for the service? Cloud-based applications, while less expensive than many conventional websites and applications, still have an expense associated with them. Charging patients directly for usage may discourage participation. Charging doctors directly for usage may do the same. Perhaps the best solution is for larger entities such as charitable, government, educational, or research groups to sponsor and pay for the usage of such a solution.

Is the system really secure? So long as no personally identifying data is stored in the cloud database, the weak points of this system will be at the "end-points". That is, with the physician's own local data storage, or the patient's own desktop or mobile device. This is no different than the current state of patient privacy issues, so this solution would not add any new security concerns to be addressed that are related to patient privacy.

Is the subjective data actually useful? After all, one patient may have a very different tolerance to pain than another, or interpret a "pain scale" differently. With the flexibility of how much information is provided and how frequent it is logged is an intentional attribute of the proposed system, it can complicate the generalization of data mined by third parties or physicians with multiple patients. While it is true that subjective data intuitively makes it difficult to make general statements, it can certainly help individual physicians evaluate and address their care for individual patients. Advanced data mining techniques could be developed to detect patterns even in the subjective data. Objective data such as medication dosage and the frequency at which it is administered can, of course, be measured more easily. Ultimately, the more data that is available, subjective or not, the more likely that patterns can be discovered and insights into useful changes in healthcare could potentially be harvested.

\section{CURRENT AND FUTURE WORK}

Work has begun on producing a prototype, with initial testing tentatively planned for late 2011. As part of a National Science Foundation program [18], students from Florida Atlantic University in Boca Raton, Florida, and collaborators from Tsinghua University and IBM China Research Laboratories in Beijing, China have already begun working on the beginning stages of the proposed solution. The first phase was completed in July, 2010 when a completely cloud-based implementation of a website and database was deployed using Amazon's Elastic Cloud (EC2) platform [19] using an opensource e-commerce software system developed Rice University entitled "Rice University Bidding Information System" (RUBIS) [20]. This software and platform was chosen to prove that a stable, user-accessible system could be deployed into the cloud and accessed by any user with a web browser, whether desktop-based or with mobile devices.

Further work was completed during the same period related to the provisioning of resources for such a system. A mathematic method was developed and proposed to handle the scaling of virtual resources required of a cloud-based system based upon demand of use of the software system. This work was accomplished with the collaboration of Tsinghua 
University and IBM China Research Labs in Beijing, using the RUBIS implementation to benchmark and test results. A paper describing this work has been submitted to the International Journal of Web Services Research and acceptance is pending at the time of this paper.

These first two phases were tested and helped develop the base platform that would be required for a healthcare-related implementation as described by this paper. The cloud implementation of the software system would need to be a stable and scalable environment that is capable of handling high usage loads if the system were to be widely adopted by patients and physicians worldwide. The software application and database layer would likewise need to be stable and accessible at all times from any location and using virtually any device. Ease-of-use of the software interface was also being tested with the implementation of the RUBIS system.

The next stage of the project proposed by this paper is to replace the e-commerce system model with the proposed health-care related system described earlier. Subsequent work may include building specific applications for a variety of mobile devices (Android-based devices, iPhone, Blackberry, et c) to improve the ease-of-use and popularity of the solution. Future work may also include proposals and demonstrations of the modification of wearable device technology (Holter monitors, blood glucose monitors, etc) to transmit data on behalf of the patient, augmenting the data logged personally by the patient. This could provide an increasingly complete picture of patient progress, further improving the effectiveness of this type of solution.

\section{CONCLUSION}

Today, mobile computing un-tethers the user from wired desktop computers and mainframes, while offering attractively smaller and more nimble device than laptops and desktops. Cloud-based computing goes even further by reducing or consolidating the infrastructure needed for the websites and data storage areas that these mobile devices access [21]. Patients no longer need to be in close proximity to healthcare providers or facilities. While technical advances continue, and devices become more ubiquitous and less expensive [22], there is room for even more improvement in the ability to assure the health and well-being of people all over the world. This may be most profoundly effective in nations with large rural or poor populations such as China and India [23][24].

The software solution proposed by this paper could have exciting and profound impact on the area of healthcare that is related to patients recently discharged from hospitals or clinics, patients in poor and rural areas of the world, and even the general public [25]. Patient-centric health data provided by patients could prove to add a wealth of new knowledge that we have never been able to analyze or act upon before. Suffering from pain, drug reactions, over- or under-prescribing of medications, wound care, physical therapy regimens or any number of other details currently missing from physician records could change how physicians treat their patients. Third party research organizations could potentially mine anonymous data and find patterns that allow physicians around the world to modify the treatment of patients in a positive way. This could be yet another novel way to leverage the growing power of social, mobile, cloud-based computing for the greater good of people worldwide, perhaps allowing us to "tweet" our way to better healthcare.

\section{REFERENCES}

[1] Hameed, K. "The Application of Mobile Computing and Technology to Health Care Services." Telematics and Informatics 20.2 (2003): 99-106.

[2] Friedman, Dick. "Mobile Computing." Electrical Wholesaling. April 1, 2006. Online at http://ewweb.com/software/electric_mobile_computing/ Last accessed on February 24, 2011.

[3] O'Mara, Deborah L. "Data Streams \& Digital Dreams." Security Dealer \& Integrator. Jan 19, 2010. Online at http://www.securityinfowatch.com/Residential+Focus/data-streams-anddigital-dreams. Last accessed on February 24, 2011.

[4] "Two So. Cal Eateries Add Mobile Order Processing Tools." Nation's Restaurant News. December 17, 2007. Online at http://www.nrn.com/article/two-so-calif-eateries-add-mobile-orderprocessing-tools. Last accessed on February 24, 1011.

[5] Waxer, Cindy. "How UPS Is Scanning and Printing Its Way to Savings" CIO.com. July 24 . Online at http://www.cio.com/article/498056/How_UPS_Is_Scanning_and_Printin g_Its_Way_to_Savings. Last accessed on Febrary 24, 2011.

[6] Howard, Courtney. "Warfighters on the Digital Battlefield Require Robust Information Technology for Secure, Reliable, Real-time Access to Mission-critical Information." Military \& Aerospace Electronics. June 16,2010 . Online at http://www.militaryaerospace.com/index/display/articledisplay/3299990254/articles/military-aerospace-electronics/exclusivecontent/2010/6/warfighters-on_the.html . Last accessed on February 24, 2011.

[7] Umedo, T., T. Higashino, C. K. Toh, and T. Isu. "An IntervehicularCommunication Protocol for Distributed Detection of Dangerous Vehicles." IEEE Transactions on Vehicular Technology 59.2 (2010): 627.

[8] Eileen Colkin Cuneo. "Mobile Care. " InformationWeek. April 1, 2004. Online at

http://www.informationweek.com/news/showArticle.jhtml?articleID=17 701265. Last acessed February 24, 2011.

[9] Lang, Kathleen. "MedQuist to Showcase Next Generation of Mobile Web Dictation at HIMSS 2008." MedQuist. February 20, 2008. Online at

http://www.medquist.com/Portals/0/news\%20releases/PhysAssist\%20IQ \%20release-FINAL.pdf. Last accessed on February 24, 2011.

[10] Renard, E. "Implantable Glucose Sensors for Diabetes Monitoring." Minimally Invasive Therapy \& Allied Technologies 13.2 (2004): 78-86.

[11] Ganapathy, Krishnan, and Aditi Ravindra. "Telemedicine in India: the Apollo Story."Telemedicine and E-health 15.16 (2009): 576.

[12] "WebMD Launches New Version of Free Mobile Application for Physicians". PR Newswire. February 16, 2010. Online at http://www.prnewswire.com/news-releases/webmd-launches-newversion-of-free-mobile-application-for-physicians-84470037.html. Last accessed February 24, 2011.

[13] Huang, Shihong; Tripathi, Shubhang; Pandya, Abhijit; Hsu, Sam; and Parker, Marilyn: "Knowledge-based Evaluation of Nursing Care Practice." Proceedings of the 2009 IEEE International Conference on Information Reuse and Integration (IRI 2009: August 10 - 12, 2009, Las Vegas, Nevada). pp. 171-174.

[14] Huang, Shihong; Chinchanikar, Suchartita; Pandya, Abhijit; Hsu, Sam; and Parker, Marilyn: "Automated Nursing Knowledge Management Using Indexing." Proceedings of the 21st International Conference on Software Engineering and Knowledge Engineering (SEKE'2009: July 1 -3, 2009; Boston, MA). pp. 93-96.

[15] "Amazon EC2 SLA." Amazon Web Services. October 23, 2008. Online at http://aws.amazon.com/ec2-sla/. Last accessed February 24, 2011. 
[16] "Number of Cell Phones Worldwide Hits 4.6B." CBS News Business. February 15, 2010. Online at http://www.cbsnews.com/stories/2010/02/15/business/main6209772.sht ml. Last accessed February 24, 2011.

[17] Rosenberg, Matt. "Current World Population.” About.com. February 1, 2011. Found online at

http://geography.about.com/od/obtainpopulationdata/a/worldpopulation. htm. Last accessed February 28, 2011.

[18] "A Global Living Laboratory for Cyberinfrastructure Application Enablement." PIRE: Partnerships for International Research and Education. November 1, 2010. Online at http://pire.fiu.edu/. Las accessed February 24, 2011.

[19] "Amazon Elastic Compute Cloud (Amazon EC2)." Amazon Web Services. November 1, 2010. Online at http://aws.amazon.com/ec2/. Last accessed February 24, 2011.

[20] ] "RUBiS: Rice University Bidding System.". February 5, 2005. Online at http://cs.nyu.edu/ totok/professional/software/rubis/rubis.html. Last accessed February 24, 2011.

[21] Di Costanzo, Alexandre, Marcos Dias De Assuncao, and Rajkumar Buyya. "Harnessing Cloud Technologies for a Virtualized Distributed
Computing Infrastructure." IEEE Internet Computing 13.5 (2009): 2433.

[22] Scholtz, Jean. "Ubiquitous Computing Goes Mobile." ACM SIGMOBILE Mobile Computing and Communications Review 5.3 (2001): 32-38.

[23] "Improving Healthcare for China's Poor." The George Institute for Global Health. Online at http://www.georgeinstitute.org/globalhealth/improving-healthcare-poor-rural-communities-china. Last accessed on February 24, 2011.

[24] "India: The Crisis in Rural Health Care." Brookings. January 24, 2008. Online at

http://www.brookings.edu/opinions/2008/0124_health_care_panagariya. aspx. Last accessed on Febrary 24, 2011.

[25] Kunze, C., Grossman, U., Stork, W., Muller-Glaser, KD. "Application of Ubiquitous Computing in Personal Health Monitoring Systems." October 23, 2009. Online at http://www.reference-

global.com/doi/abs/10.1515/bmte.2002.47.s1a.360. Last accessed February 24, 2011. 